\title{
Optimal pricing and quality policy considering consumer learning behaviors in online marketplaces
}

\author{
WANG Xuehong \\ Shandong Yingcai University, Shandong, Jinan, 250104, China \\ 495656305@qq.com
}

Keywords: online marketplaces; consumer learning behaviors; pricing; quality policy

\begin{abstract}
Consumers always learn from the information about the historical sale records and the feedbacks from other buyers with the nature of the online marketplace, which refers to its virtual property, and then make their decisions. This kind of consumers' behaviors that learning from other buyers proposes a challenge for corporations (manufacturers) to determine the products' quality level and prices. Based on that, we investigate the manufacturers' strategies of pricing and quality policy by establishing a two-period supply chain model when considering the effect of customers learning behaviors. And then we compare these equilibriums with the results in another scenario that the manufacturer does not consider the consumers learning behaviors. Finally, we further examine the multi-period case where the manufacturer has to decide product level and prices of each period.
\end{abstract}

\section{Introduction}

More and more enterprises through the network channels to direct sales the products to consumers, such as HP, IBM, Apple and other large manufacturing enterprises which have the network direct marketing channels. At the same time, consumers are more frequently purchase products through the network channels. But products could not be directly observed and experience, consumers generally need to observe other consumer buying behavior, enterprise credit evaluation and information such as product sales records after make buying decisions.

Therefore, in the period of the network market products in the sales process, manufacturers how to balance the quality of the products and price to avoid the impact of consumer learning behavior, and maximize the profit of their own is a question worth pondering. Under the premise of the purpose of this paper is to consider the consumer learning behavior, by building a game model, research manufacturers in multiphase product sales in the process of product quality and pricing decision problems.

At present, enterprises under the market environment of network quality and pricing strategy research mainly focused on the specific product pricing, dynamic pricing and double channel pricing, etc. Such as Horsky, Krishnan for durable goods in network market pricing strategy problems are studied, found that due to the learning curve effect exist in the production, so prices the optimal strategy for manufacturers. Cheng yan studied is seasonal, fashion model under the network retail product line dynamic pricing problem, this paper proposes a verified feasibility of the algorithm and simulation analysis. The research methods of game theory, optimization from the Angle of quantitative product pricing problem is studied under the network environment, but also under the scope limited to the special properties of product pricing, lack of the study of consumer behavior factors.

Because of this, this article will explore the network market environment when there is consumer learning behavior and the early stage of the product sales volume has influence on current demand, manufacturers product quality and pricing decision problems, the conclusion can provide reference for enterprise actual operation decision. 


\section{Problem description and the demand model}

This paper consider a also responsible for product production and distribution of oligarch manufacturer with two layers of the supply chain composed of consumers, the manufacturers need to early in the price of product quality and two sales cycle to make decisions. In network market, the consumer learning behavior, namely the second phase of the consumer will be according to the first issue of product sales to judge the quality of the products and make a purchase decision, therefore, manufacturers need to consider the first issue of product quality and the price of two products demand.

According to Moorthy and Örsdemir, assumes that the network market, consumers are heterogeneous in their willingness to pay (WTP) for unit products subject to the uniform distribution of $[0,1]$. For a willingness to pay for the consumer, the quality level is willing to pay a price for $\theta s$, this means that when other condition is constant, high quality products for consumers in preference above preference for low quality products. Given initial (first phase) product prices $p_{1}$, the consumer surplus for consumers to buy the unit products $U_{1}=\theta s-p_{1}$, further to demand function $q_{1}=1-p_{1} / s$.Similarly, assuming that the second phase of the product sales price for the $p_{2}$, is the second phase of the consumers to buy in per unit product of consumer surplus for $U_{2}=\theta s^{\prime}-p_{2}$ or $U_{2}=\theta\left(1+\delta q_{1}\right) s-p_{2}$, therefore, the second phase of the product demand for $q_{2}=1-p_{2} /\left[\left(1+\delta q_{1}\right) s\right]$.

Due to product quality influences the market demand for products and the early stage of the product sales volume has a positive influence on the current demand, manufacturers hope to improve the product quality to increase the product demand, at the same time, improve the quality of our products often have to spend more cost. According to document [19], [20], assuming that the quality of the products and meet between the total cost: $\mathrm{M}$, which is suitable for scale parameter $C=\beta s^{2}$. The total cost of $\beta>0$ is about the quality of the products of the second order continuous differentiable function. In addition, assuming that the cost of production per unit product for $c_{0}$, transport costs for $C_{1}$. Convenience for subsequent calculations, assuming that the production cost and transport cost is zero.

\section{Model building}

To illustrate consumer learning behavior influence on manufacturers product quality and pricing decision, this paper studies the manufacturer does not consider the optimal pricing when consumer learning behavior and decision quality, namely the first phase of the product sales for the second phase of the customer perceived quality influence is zero, manufacturers in both product quality and pricing decisions within the sales cycle. The conclusion will be discussed later when consumer learning behavior manufacturers provide comparison standard optimal decision.

\subsection{Case 1 (regardless of consumer learning behavior)}

When the manufacturer does not consider factors influencing consumer learning behavior, namely the first phase of the product sales for the second phase of the customer perceived quality influence is zero, the manufacturer's profit function as :

$$
\begin{aligned}
\pi^{N C}= & \left(1-p_{1}^{N C} / s^{N C}\right) p_{1}^{N C}+\left(1-p_{2}^{N C} / s^{N C}\right) p_{2}^{N C} \\
& -\beta\left(s^{N C}\right)^{2}
\end{aligned}
$$

Among them: superscript "NC" said manufacturers do not consider the situation of consumer learning behavior.

By type (1), when the maker doesn't consider the effect of consumer learning behavior its optimal decision can be made of the nature of the 1 . 
Nature 1: when manufacturers do not consider when consumer learning behavior, the optimal quality and pricing decision I and II, respectively.

Proof: by reverse method, given the first phase of $s^{N C}$ and price $p^{N C}$, product quality by type (1) available manufacturers in the second phase of the optimal pricing for $p_{2}^{N C^{*}}=s^{N C} / 2$. $p_{2}^{N C^{*}}$ generations into (1) can be further obtained, manufacturers in the first phase of the optimal pricing and quality decision $p_{1}^{N C^{*}}=1 /(8 \beta)$ and $s^{N C^{*}}=1 /(4 \beta)$, respectively.

So when the manufacturer doesn't consider the effect of consumer learning behavior factor, the manufacturer's optimal decision for $p_{1}^{N^{*}}=p_{2}^{N^{*}}=1 /(8 \beta)$ and $s^{N^{*}}=1 /(4 \beta)$.

Properties of 1 shows that when the manufacturer does not consider network learning behavior of consumers in the market, the same two sales during the period of product pricing. In addition, the manufacturer, the optimal pricing and product quality decision is inversely proportional to the coefficient of size $\beta$. This is because when the scale coefficient $\beta$ is larger, the production of a certain quality of product of the total cost is high, the manufacturer will choose to produce low quality products, thus reduce the product price.

In addition, by the nature of the 1 available of the manufacturer's profits under the conditions of the case $1 \pi^{N C^{*}}=1 /(16 \beta)$.

\subsection{Case 2 (consider consumer learning behavior)}

Below manufacturers consider when consumer learning behavior exist in the network market, the manufacturer's product quality and pricing decisions are analyzed. According to the previous analysis, in this case the manufacturer's profit function for

$$
\begin{aligned}
\pi^{C}= & \left(1-p_{1}^{C} / s^{C}\right) p_{1}^{C}+ \\
& \left(1-\frac{p_{2}^{C}}{\left[1+\delta\left(1-p_{1}^{C} / s^{C}\right)\right] s^{C}}\right) p_{2}^{C}-\beta\left(s^{C}\right)^{2}
\end{aligned}
$$

Among them: superscript "C" said manufacturers consider consumer learning behavior.

According to the reverse method, can be introduced under the case 2 manufacturer's optimal decision, the result can be made of nature of 2 .

Properties 2, in the manufacturers under the condition of considering network in the market of consumer learning behavior, when the two sales cycle are manufacturers of optimal decision $p_{1}^{C^{*}}=\frac{4-\delta}{8}\left[\frac{1}{4 \beta}+\frac{\delta^{2}+8 \delta}{128 \beta}\right], \quad s^{C^{*}}=\frac{1}{4 \beta}+\frac{\delta^{2}+8 \delta}{128 \beta}$ and $p_{2}^{C^{*}}=\frac{4+(\delta+2)^{2}}{16}\left[\frac{1}{4 \beta}+\frac{\delta^{2}+8 \delta}{128 \beta}\right]$.

Proof: given the first term of product quality and product price, the manufacturer in the second phase of the optimal pricing can be converted into the following optimization problem:

$$
\begin{aligned}
& \max _{p_{2}^{C}} \pi^{C}\left(p_{2}^{C} \mid p_{1}^{C}, s^{C}\right)=\left(1-p_{1}^{C} / s^{C}\right) p_{1}^{C}+ \\
& \left(1-\frac{p_{2}^{C}}{\left[1+\delta\left(1-p_{1}^{C} / s^{C}\right)\right] s^{C}}\right) p_{2}^{C}-\beta\left(s^{C}\right)^{2} \\
& \text { s.t. } p_{2}^{C} \geq 0 \\
& p_{2}^{C}=\frac{\left[1+\delta\left(1-p_{1}^{C} / s^{C}\right)\right] s^{C}}{2} \\
& \max _{p_{1}^{C}, s^{C}} \pi^{C}\left(p_{1}^{C}, s^{C}\right)=\left(1-p_{1}^{C} / s^{C}\right) p_{1}^{C}+ \\
& \frac{\left[1+\delta\left(1-p_{1}^{C} / s^{C}\right)\right] s^{C}}{4}-\beta\left(s^{C}\right)^{2} \\
& \text { s.t. } p_{1}^{C}, s^{C} \geq 0 \\
& s^{C^{*}}=\frac{1}{4 \beta}+\frac{\delta^{2}+8 \delta}{128 \beta} \text { and } p_{1}^{C^{*}}=\frac{4-\delta}{8}\left[\frac{1}{4 \beta}+\frac{\delta^{2}+8 \delta}{128 \beta}\right]
\end{aligned}
$$




$$
\begin{aligned}
& \text { Becauce } p_{1}^{C} \geq 0, \text { so } 4 \geq \delta, \text { To plug in type } p_{1}^{C^{*}} \text { and } s^{C^{*}} \quad(4), \text { will be } \\
& p_{2}^{C^{*}}=\frac{4+(\delta+2)^{2}}{16}\left[\frac{1}{4 \beta}+\frac{\delta^{2}+8 \delta}{128 \beta}\right] \text { 。 } \\
& \text { If } \delta>4, p_{1}^{C^{*}}=0 \text {,(3)will be } s^{C^{*}}=(1+\delta) /(8 \beta) \text { and } p_{2}^{C^{*}}=(1+\delta)^{2} /(32 \beta) .
\end{aligned}
$$

\section{Numerical analysis}

First of all, the analysis parameters, and the impact on the manufacturers product quality and pricing decisions, the figure 1, 2, and 3 shows that compared with no account of situation of consumer learning behavior, in considering the situation of consumer learning behavior in network market, the manufacturer's optimal decision should be to raise the level of product quality, to lower the price of the first product sales cycle, and improve the product prices in the second period.

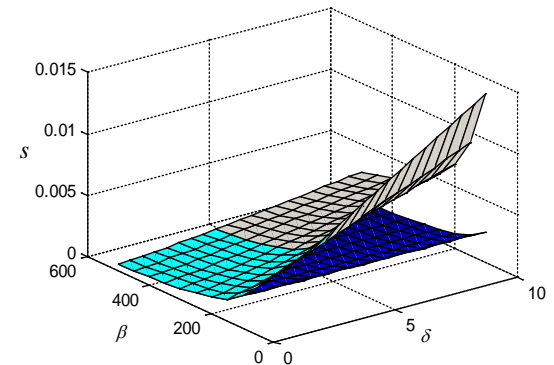

Figure 1

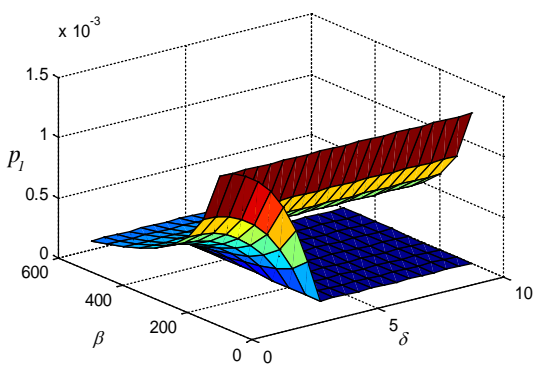

Figure 2

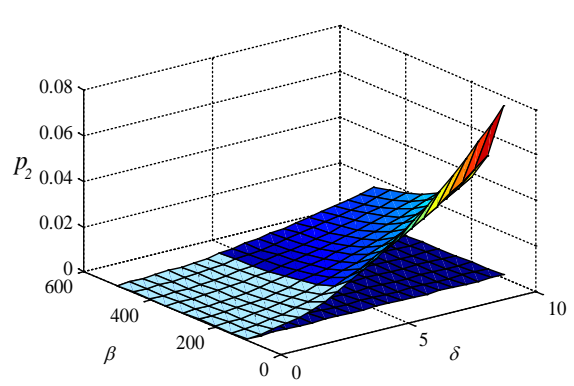

Figure 3

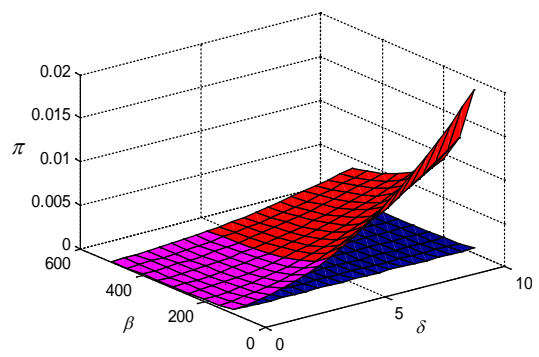

Figure 4

The figure 4 shows that when $\delta$ remains the same, with the increase of $\beta$, manufacturers gradually reduce profits. The larger the scale coefficient, improve the level of product quality the higher costs, so manufacturers lower profits.

\section{Summary}

This paper studies the consumer learning behavior in the network market makers in the network market product quality level and multiphase product pricing problems, which influence consumer learning behavior mainly for the early stage of the product sales for the current customer perceived quality has a positive effect. First, discuss in two product sales cycle manufacturer's product quality and two pricing decisions, and learn with the manufacturer does not consider the consumer behavior under the factors of product quality and pricing decision, this paper compares and analyzes found in consideration of consumer learning behavior factors in the network market, manufacturers through in the first time to take high quality and low price, high price in the second phase of the strategy, can obtain higher than does not consider the consumer learning behavior under the corresponding returns.

\section{Acknowledgments}

Project funds: The subject of Shandong YingCai University(15YCYBRW01).The subject of 
Shandong province bureau of statistics(KT16134).

\section{References}

[1] Yoo W, Lee W. Internet channel entry: a strategic analysis of mixed channel structures [J]. Marketing Science, 2011, 30(1): 29-41.

[2] Zhao Y, Yang S, Narayan V, Zhao Y. Modeling Consumer learning from online product review [J]. Marketing Science, 2013, 32(1): 153-169.

[3] Ye Q, Cheng Z J, Fang B. Learning from other buyers: the effect of purchase history records in online marketplaces [J]. Decision Support Systems, 2013 56: 502-512.

[4] Chen J, Zhang H, Sun Y. Implementing coordination contracts in a manufacturer Stackelberg dual-channel supply chain [J]. Omega, 2012, 40(5): 571-583.

[5] Xu G Y, Dan B, Zhang X M, Liu C. Coordinating a dual-channel supply chain with risk-averse under a two-way revenue sharing contract [J]. International Journal of Production Economics, 2014, 147(part A):171-179. 